\title{
QTL ANALYSIS OF FIBER COLOR AND FIBER QUALITY IN NATURALLY GREEN COLORED COTTON (Gossypium hirsutum L.)
}

\author{
Devrim Semizer CUMING ${ }^{*} \dagger$, Filiz ALTAN ${ }^{2}$, Huseyin AKDEMIR ${ }^{3}$, Muzaffer TOSUN ${ }^{4}$, \\ Aynur GUREL ${ }^{l}$, Bahattin TANYOLAC ${ }^{l}$ * \\ ${ }^{1}$ Ege University, Faculty of Engineering, Department of Bioengineering, Izmir, TURKEY \\ ${ }^{2}$ Mugla Sitki Kocman University, Faculty of Science, Department of Biology, Mugla, TURKEY \\ ${ }^{3}$ Ege University, Odemis Vocational Training School, Izmir, TURKEY \\ ${ }^{4}$ Ege University, Faculty of Agriculture, Department of Field Crops, Izmir, TURKEY \\ †Present Address: Gottingen University, Department of Forest Genetics and Tree Breeding, GERMANY \\ Corresponding authors:dsemize@forst.uni-goettingen.de,bahattin.tanyolac@ege.edu.tr
}

Received: 21.10.2014

\begin{abstract}
Improving fiber quality of naturally colored cotton has become more important in recent years due to changes in the spinning technology and consumer demand. The identification of quantitative trait loci (QTL) closely linked to the fiber quality traits can allow marker assisted selection (MAS) which leads us to future's improved cotton cultivars. In this study, we performed genetic mapping on $\mathrm{F}_{2}$ population of $G$. hirsutum (Yesil $\times$ Nazilli 84) intraspecific cross, and identified QTL for fiber color parameters $(L, a, b, \Delta L, \Delta a, \Delta b, \Delta E)$ and fiber quality traits (fiber length, uniformity and elongation). The resulting genetic linkage map comprised of 123 amplified fragment length polymorphism (AFLP) markers and 27 linkage groups (LGs), covering $2068.5 \mathrm{cM}$ with an average distance of $16.8 \mathrm{cM}$ between two markers. Using single marker analysis a total of 43 QTL for fiber color parameters and fiber quality traits were identified, including four for fiber length, two for fiber uniformity, two for fiber elongation, five for $L$, four for $a$, four for $b$, eight for $\Delta L$, four for $\Delta a$, four for $\Delta b$ and six for $\Delta E$. The identified QTL for fiber color parameters and fiber quality traits explained between $7.8 \%$ and $14.6 \%$ and between $5.9 \%$ and $14.7 \%$ of the phenotypic variation, respectively. Additionally, recombinant individuals having green fiber color together with long fiber length, high fiber uniformity and elongation were obtained in the segregating population. These individuals can be used to develop new cotton varieties in the future.
\end{abstract}

Keywords: AFLP, genetic linkage mapping, green cotton fiber, G. hirsutum L., QTL.

\section{INTRODUCTION}

Being the world's most widely cultivated fiber crop, and the most important natural textile fiber, cotton has significant importance to the world economy. Although the demand for naturally colored cotton is increasing every year, its cultivation has not increased accordingly. This is largely because naturally colored cotton is often associated with low yield and poor fiber quality since there is a negative correlation between natural pigmentation and yield and fiber quality that is hard to break by traditional crossing and selection (Zhao et al., 2009). Faster spinning systems, on the other hand, require higher fiber quality.

Increasing yield and improving fiber quality have always been the main goals of cotton breeding. However, most of these traits are quantitative traits that are controlled by many genes interacting with their environment (Ulloa et al., 2007; Zhao et al., 2009). Using molecular markers closely linked to the QTL is a faster and more effective approach for pyramiding desired traits into a genotype. For this reason, molecular linkage map construction using neutral genetic markers has been recognized as an essential tool for plant molecular breeding (Wu et al., 2009).

For over a hundred year, the genetic background of naturally colored cotton has been the subject of several studies, with substantively varying results. According to Fletcher's (1907) study on Mendelian heredity in cotton, color is dominant over white. Some early studies stated that the lint color is determined by a group of genes situated at six loci (Lc1-Lc6) (Silow, 1944; Endrizzi and Kohel, 1966; Kohel, 1985). However, Richmond (1943) reported that in Texas green and brown lint, each color was controlled by a single incompletely dominant gene. In Murthy (2001), it was defined that the gene(s) for lint color is often found to be pleotropic, i.e. controlling more than one trait. Later, researchers (Shi et al., 2002; Fan et al., 2009; Wu et al., 2010) reported that both brown and 
green colored lint are controlled by one pair of genes incomplete in dominance on nonhomologous chromosomes, respectively. Li et al. (2004) and Zhan et al. (2008) also reported that the fiber color of brown cotton was controlled by a single incomplete dominant gene. Zhu et al. (2003), on the other hand, indicated that the cotton fiber color trait was a quantitative trait controlled by polygene. Dutt et al. (2004) reported that fiber color was controlled by a single dominant gene. Feng et al. (2010) claimed that the genetic variation of the brown lint in upland cotton is mainly derived from additive and dominant effects, in which the additive effect is predominant and the different fiber colors in $F_{1}$ generation resulted from the different additive effects of different varieties or lines.

In the current study, in order to understand the gene(s) controlling the fiber color and the fiber quality traits we crossed two upland cotton cultivars displaying different fiber color and fiber quality traits. We then constructed a genetic linkage map using $\mathrm{F}_{2}$ segregating population and finally identified the genetic markers linked to the fiber color and the fiber quality traits by conducting QTL analysis.

\section{MATERIALS AND METHODS}

\section{Plant materials}

Two upland cotton cultivars with different fiber color and contrasting fiber quality traits were used to develop an intraspecific $\mathrm{F}_{2}$ mapping population (Table 1 and 2). The female parent was G. hirsutum cv. Yesil with green fiber color and the male parent was $G$. hirsutum cv. Nazilli-84 with white fiber color. The two parents were crossed in the field experiment located in the Odemis Vocational Training School fields and the resulting $F_{1}$ hybrid seeds were grown at FIDAS, Inc. (Izmir, Turkey) until each of the seedlings were $15 \mathrm{~cm}$ in height. The seedlings were then transferred to the greenhouse at the Department of Bioengineering and planted there in the pots $30 \times 30 \mathrm{~cm}$ in size and selfed to form $F_{2}$ population. A total of $94 F_{2}$ individuals were used to construct the linkage map.

Table 1. Phenotypic variation of the three fiber quality traits in $94 \mathrm{~F}_{2}$ individuals and the parent lines Yesil and Nazilli-84

\begin{tabular}{lcccccccc}
\hline \multirow{2}{*}{ Fiber quality trait } & \multicolumn{3}{c}{ Parent lines } & \multicolumn{5}{c}{ Variation in the population } \\
\cline { 2 - 9 } & Yesil & Nazilli-84 & Mean & Min. & Max. & Std. Dev. & Skewness & Kurtosis \\
\hline Length (mm) & 18.5 & 27.6 & 26.3 & 16.9 & 29.5 & 1.49 & -0.53 & 19.77 \\
Uniformity (\%) & 77 & 85 & 83.04 & 77.4 & 87 & 1.71 & -0.26 & 0.24 \\
Elongation (\%) & 4.5 & 7.5 & 6.67 & 4.4 & 9.5 & 0.73 & 0.02 & 3.43 \\
\hline
\end{tabular}

Table 2. Phenotypic variation of the fiber color parameters in $94 F_{2}$ individuals and the parent lines Yesil and Nazilli- 84

\begin{tabular}{lcccccccc}
\hline Fiber color & \multicolumn{3}{c}{ Parent lines } & \multicolumn{5}{c}{ Variation in the population } \\
\cline { 2 - 8 } parameter $^{\dagger}$ & Yesil & Nazilli-84 & Mean & Min. & Max. & Std. Dev. & Skewness & Kurtosis \\
\hline $\mathrm{L}$ & 57.59 & 85.97 & 69.79 & 59.63 & 87.37 & 4.06 & 1.49 & 5.99 \\
$\mathrm{a}$ & 0.58 & 3.18 & -0.35 & -3.21 & 10.01 & 1.76 & 4.07 & 22.3 \\
$\mathrm{~b}$ & 20.4 & 9.71 & 16.11 & 3.45 & 24.52 & 2.69 & -1.48 & 8.31 \\
$\Delta \mathrm{L}$ & -35.6 & -7.2 & -23.4 & -33.56 & -5.83 & 4.03 & 1.51 & 6.14 \\
$\Delta \mathrm{a}$ & 2.9 & 0.29 & -0.63 & -3.49 & 9.73 & 1.75 & 4.11 & 22.65 \\
$\Delta \mathrm{b}$ & 22.55 & 11.87 & 18.26 & 5.61 & 26.67 & 2.69 & -1.47 & 8.29 \\
$\Delta \mathrm{E}$ & 42.28 & 13.96 & 29.88 & 8.12 & 44.07 & 4.57 & -1.84 & 9.35 \\
\hline
\end{tabular}

†Fiber color parameters: $\mathrm{L}$ indicates color brightness $(\mathrm{L}=0$ is black and $\mathrm{L}=100$ is white); a indicates red-green ( + is red and - is green); $b$ indicates yellow-blue ( + is yellow and - is blue); $\Delta \mathrm{L}$ indicates total black-white color change; $\Delta \mathrm{a}$ indicates total red-green color change; $\Delta \mathrm{b}$ indicates total yellow-blue color change; $\Delta \mathrm{E}$ indicates total color change independent from each other.

\section{Trait phenotypic analysis}

Fiber colors of the parents and each $\mathrm{F}_{2}$ individual were quantified as $L, a, b, \Delta L, \Delta a, \Delta b, \Delta E$ parameters using Minolta C400 Chromameter (Konica Minolta Holdings, Inc., Tokyo, Japan). Fiber length, fiber uniformity and fiber elongation were measured using USTER Fiber Analysis System (USTER Technologies, Inc., Knoxville, TN, USA). Basic statistical calculations were conducted using Microsoft Excel, $\chi^{2}$ tests and correlations were performed using TARIST software (Acikgoz et al., 1994).

\section{Marker analysis}

Genomic DNA was extracted from young and fully expanded frozen leaves using Doyle and Doyle (1987) with a minor modification (precipitation in $\mathrm{EtOH}$ for 3 times). The AFLP procedure was followed according to the manufacturer's recommendations (LI-COR NEN Model 4300 DNA Analysis System, LI-COR Biosciences, Lincoln, NE, USA) using AFLP 2-Dye Selective Amplification Kit (Cat. No. 830-06197). Briefly, 200ng of genomic DNA from each genotype was digested with EcoRI and MseI, followed by ligation of the adapters to the restriction fragments. A primer pair containing the EcoRI- and MseI-adapter sequences with one selective nucleotide was used for preamplification. A total of 64 primer pairs from a combination of EcoRI and MseI primers were used for the selective amplifications performed using primer pairs containing three selective nucleotides extension. All of the EcoRI primers were IRDye fluorescent labeled, four of which were IRDye 700-, and the other four were IRDye 800-labeled. A selective amplification was carried out using one MseI with two IRDye labeled EcoRI primers. PCR 
amplifications were performed in a PTC-225 DNA Engine Tetrad Thermal Cycler (MJ Research, Inc., Watertown, MA, USA). The amplified fragments were separated and visualized on polyacrylamide gels (8\%) using LI-COR 4300 DNA Analyzer.

\section{Linkage analysis and map construction}

AFLP markers were first screened in the parents and $\mathrm{F}_{1}$ plants to detect the polymorphisms, then the polymorphic markers were further used for genotyping the $\mathrm{F}_{2}$ individuals. Informative bands were scored as " $\mathrm{A}$ " or " $\mathrm{B}$ " regarding the resemblances to the female or male parent genome, while non-informative bands were scored as "-". Linkage analysis was carried out using MapMaker 3.0 (Lander et al., 1987) with logarithm of odds (LOD) threshold of 3.0 and a recombination frequency of 0.50 to provide evident linkage. The Kosambi map function (Kosambi, 1944) was used to convert the recombination frequencies to the map distances. The graphic representation of the linkage groups was created at the end of the linkage analysis.

\section{QTL analysis}

Single marker analysis was employed to determine the effect of each marker on each trait measured in $F_{2}$ segregating population using QGene 4.0 (Nelson, 1997). A stringent LOD threshold of 3.0 was used to declare significant QTL for fiber color and fiber quality traits. Meanwhile, an LOD score value less than 2.0 was also used to detect any putative QTL.

\section{RESULTS}

\section{Trait phenotypic analysis}

The phenotypic variations of the three fiber quality traits and seven fiber color parameters were summarized in Table 1 and Table 2, respectively. Transgressive segregations were observed in $F_{2}$ population in all fiber quality traits and fiber color parameters except for $\Delta L$. The frequency distribution of each parameter in $F_{2}$ individuals showed typical quantitative variation (data not shown). All variables fitted a normal distribution and none were transformed in further analyses. The $\chi^{2}$ test results for fiber color and fiber elongation were given in Table 3 and 4 , respectively. The test results indicated that green fiber color was controlled by a single dominant gene (Table 3), while fiber elongation showed digenic inheritance with an epistatic interaction as duplicate gene action (Table 4). On the other hand, the $\chi^{2}$ test results suggested a polygenic inheritance for fiber length, fiber uniformity and all seven color parameters since the test results did not agree with any segregation. However, they indeed showed transgressive segregations regarding lower and higher values (Table 1 and 2).

Table 3. $\chi^{2}$ test result for fiber color in the $\mathrm{F}_{2}$ population

\begin{tabular}{llc}
\hline & $\chi^{2}$ & $\mathbf{p ~ ( 5 \% )}$ \\
\hline White & 0.025 & \\
Green & 0.0086 & 3.84 \\
Total & 0.0336 & $\mathbf{p ~ ( 5 \% )}$ \\
\hline Table 4. $\chi^{2}$ test result for fiber elongation in the $\mathrm{F}_{2}$ population \\
\hline \multicolumn{3}{l}{$\chi^{\mathbf{2}}$} \\
\hline 15 & 0.29 & \\
1 & 2.7 & 3.84 \\
Total & 2.75 & \\
\hline
\end{tabular}

\section{Polymorphism level and marker genotyping}

AFLPs were first analyzed between the two parental lines and their $F_{1}$ hybrids and then in $94 F_{2}$ individuals for marker segregation tests. Of the 64 primer combinations, 26 showed polymorphisms in the segregating population. 26 AFLP primer combinations yielded a total of 240 polymorphic bands suggesting that an average of 9.2 informative AFLP per primer pair was scored.

\section{Linkage mapping}

A total of 240 AFLP markers was employed to perform linkage analysis. A hundred and twenty three markers were distributed into 27 LGs (Figure 1). While 117 markers remained unlinked, suggesting that about $51 \%$ of the total polymorphic markers contributed to the linkage map. The linkage map covered $2068.5 \mathrm{cM}$. The LGs varied from 3.2 to $183.6 \mathrm{cM}$ with the average distance about $16.8 \mathrm{cM}$ between two adjacent markers.

\section{QTL analysis}

A total of 43 QTL for fiber related traits were identified on 11 LGs based on single marker analysis. The positions and effects of QTL contributing to the fiber quality traits and fiber color parameters were summarized in Table 5 and 6 , respectively. Sixteen LGs had no associations with any QTL for these traits. Of the 43 QTL, 8 were detected for the three fiber quality traits: four for fiber length, two for fiber uniformity and two for fiber elongation. Thirty five QTLs were detected for the seven fiber color parameters: five for $L$, four for $a$, four for $b$, eight for $\Delta L$, four for $\Delta a$, four for $\Delta b$ and six for $\Delta E$. The identified QTL for the fiber quality traits and for the fiber color parameters explained 5.9 to $14.7 \%$ and 7.8 to $14.6 \%$ of the phenotypic variation, respectively. 


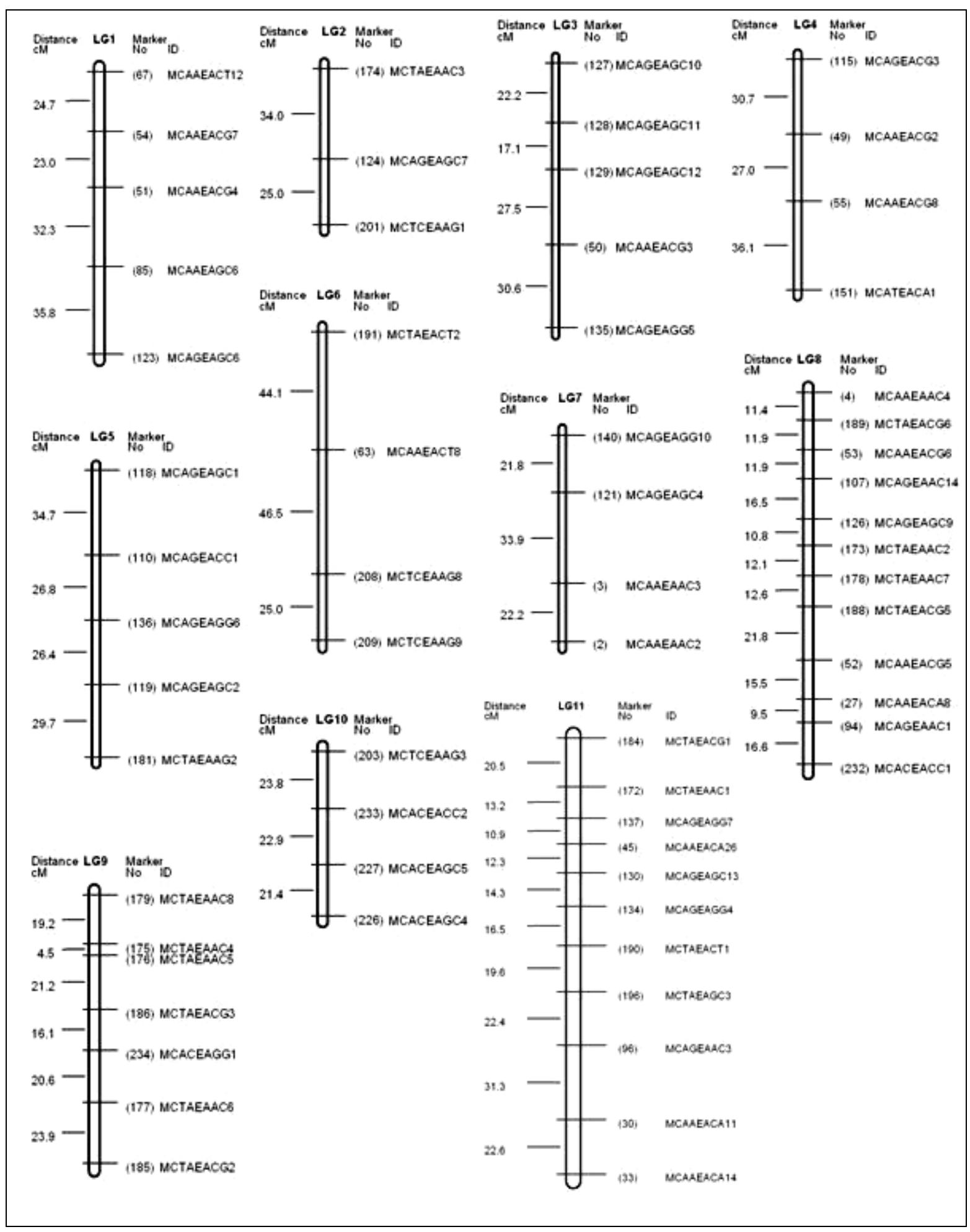

Figure 1. 27 linkage groups (LGs) resulted from linkage analysis 


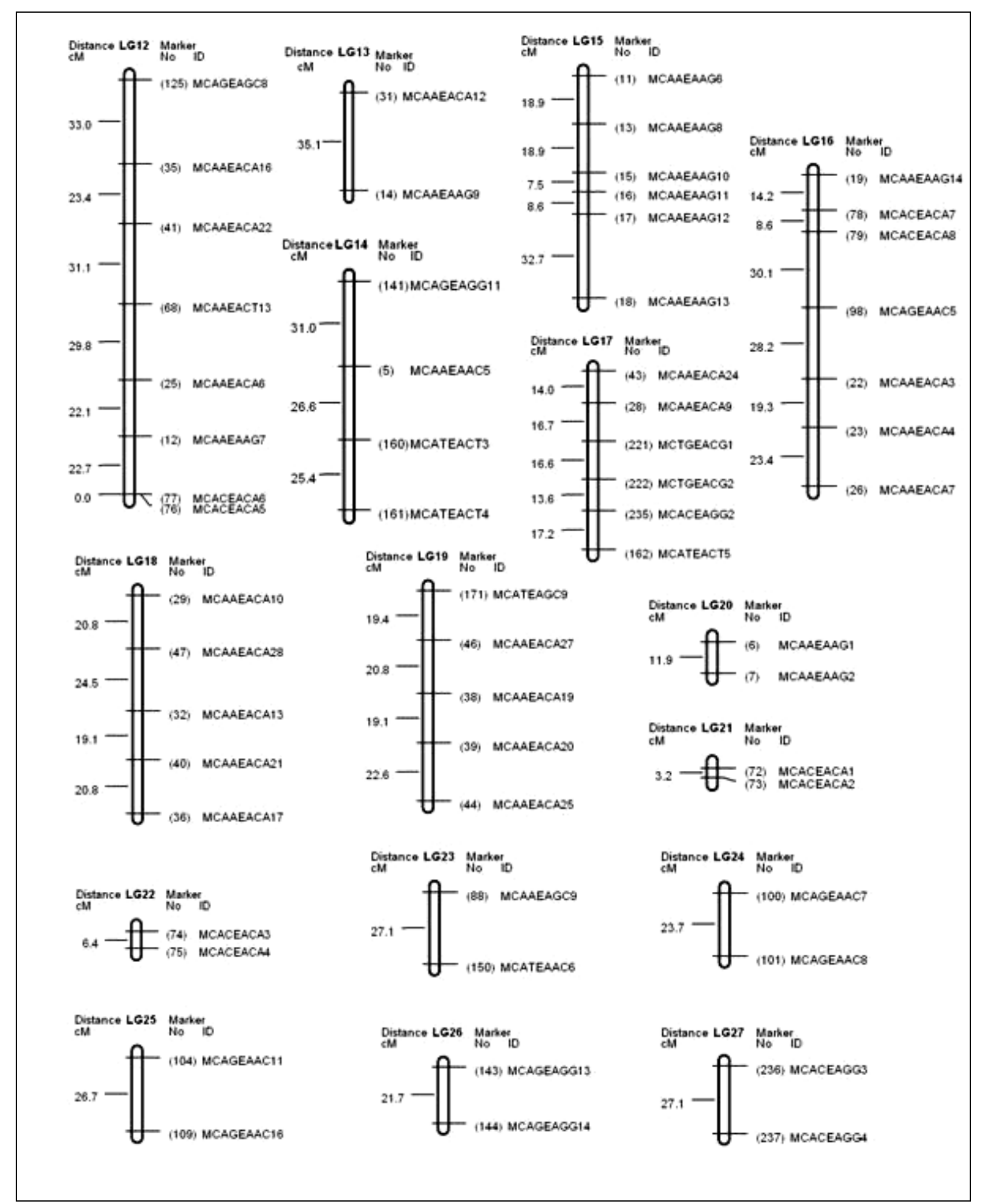

Figure 1. 27 linkage groups (LGs) resulted from linkage analysis (contd.) 


\section{DISCUSSION}

As a step toward understanding more about the naturally colored cotton genome, we first analyzed seven fiber color parameters and three fiber quality traits in the $\mathrm{F}_{2}$ individuals using the fiber quality data collected (Table 1 and 2). Fiber elongation was the most variable trait while fiber uniformity was the least variable among the individuals in the segregating population. Additionally, the color parameter " $a$ " was the most variable among the seven fiber color parameters, while $L$ was the least variable. The result of the fiber color parameter " $a$ " being the most variable trait is of particular interest since this parameter defines the color range related to green. Even though there is no similar study published on cotton fiber color to compare our results with, there have been many results reported on variations of cotton fiber quality traits in different types of populations using different parental crosses so far (Lin et al., 2005; Zhang et al., 2005; An et al., 2010). Despite the fact that the amount of variations existed for fiber length, fiber uniformity and elongation in these studies were similar to our study.

Table 5. QTL linked to the fiber quality traits

\begin{tabular}{lllccc}
\hline Fiber quality trait & QTL & Marker ID & Linkage Group & LOD & R $^{\mathbf{2}}(\boldsymbol{\%})^{\dagger}$ \\
\hline Fiber length & FL1 & MCAAEACG7 & 1 & 2.9 & 14.7 \\
& FL2 & MCAAEACT12 & 1 & 2.82 & 13.7 \\
& FL3 & MCTAEAAC2 & 8 & 1.82 & 9.4 \\
\multirow{3}{*}{ Fiber uniformity } & FL4 & MCATEACA5 & - & 1.63 & 8.6 \\
& FU1 & MCTCEAAG10 & - & 1.79 & 9.1 \\
Fiber elongation & FU2 & MCAGEAGG1 & - & 1.18 & 5.9 \\
& FE1 & MCACEAGG6 & - & 2.48 & 12.2 \\
\hline
\end{tabular}

${ }^{2}(\%)$ indicates the percentage of the phenotypic variance explained by the QTL.

In the present study we observed transgressive segregations in all of the fiber quality traits and color parameters -except for $\Delta L$ - in the $\mathrm{F}_{2}$ individuals. The transgressive segregations for fiber length and fiber elongation fell beyond both parents, while those for fiber uniformity exceeded Nazilli-84. This shows an increase in fiber uniformities in the segregating population. This result was supported by Price et al. (2001) reporting that naturally green colored cottons possess the most uniform fibers among the naturally colored cottons. Transgressive segregations exceeded Nazilli-84 in $L$, and both parents in $a, b, \Delta a, \Delta b$ and $\Delta E$ values. There are transgressive segregations reported in fiber related traits in the literature (Wang et al., 2006; Shen et al., 2007; Qin et al., 2008; Luan et al., 2009; An et al., 2010), which were consistent with our current results. It has been also demonstrated that $\mathrm{F}_{2}$ hybrids can exhibit superior performance for agronomic and fiber traits when compared with their parental lines (Gutiérrez et al., 2002).

Based on the $\chi^{2}$ test, green fiber color was controlled by a single dominant gene (Table 3 ), while all of the fiber color parameters and the fiber quality traits were controlled by multiple genes. Only fiber elongation expressed digenic inheritance with a duplicate gene action (Table 4). Despite the differences in parents and type of segregating populations, our results were comparable with the other studies. Similar to our results, both Fletcher (1907) and Dutt et al. (2004) reported that fiber color was controlled by a single dominant gene. Chaudhary et al. (2010) reported that dominancy and epistasis were effective in fiber uniformity and fiber elongation while only epistasis is effective in fiber uniformity. Subhan et al. (2003) also denoted that dominancy or epistasis was important in the inheritance of fiber length trait in $F_{2}$ population of upland cotton. However, Creatham et al. (2003) stated that both additive and dominant genetic effects control fiber elongation. Jambhale et al. (1998) revealed the existence of duplicate gene action and the role of both additive and dominant gene effects in the control of fiber traits, and selected $\mathrm{F}_{2}$ and $\mathrm{BC}$ individuals with brown and green fiber color and longer fiber. In the present study, we also obtained recombinant individuals having green fiber color and long fiber length (max. $29 \mathrm{~mm}$ ), high fiber uniformity (max. 85.9\%) and high fiber elongation (max. 7.9\%). These individuals can be regarded as assets to develop new cotton varieties in the future.

In order to identify QTL for fiber color and fiber quality traits in naturally green colored cotton, we constructed a genetic linkage map with AFLP markers using the $F_{2}$ population. The resulting map contained 27 LGs (Figure 1) and covered $2068.5 \mathrm{cM}$ with the average distance about $16.8 \mathrm{cM}$ between two markers. Based on single marker analysis, we detected a total of 43 QTL contributing to the seven fiber color parameters and three fiber quality traits (Table 5 and 6). Of 43 QTL, 8 were detected for the fiber quality traits and 35 were detected for the fiber color parameters. However, typically an LOD threshold of between 2 and 3 is required to ensure an overall false positive rate of 5\% (Lander and Botstein, 1989). Regarding the LOD values in our study, we obtained $L O D \geq 2$ with the three QTL for fiber quality traits and 17 QTL for fiber color parameters. The rest of the 23 QTL were below LOD 2. The results were nevertheless reported for all the QTL in this study. However, QTL having LOD values below the permutation based threshold should be treated cautiously. The identified QTL for the fiber quality traits explained 5.9 to 
$14.7 \%$ of the total phenotypic variation while for the fiber color parameters explained 7.8 to $14.6 \%$ of the total phenotypic variation. In spite of the fact the data in our study shows that all QTL had relatively small effects controlling less than $20 \%$ of the total phenotypic variation (Table 5 and 6), suggesting the fiber color and fiber quality traits might be controlled by minor genes. This might be, however, resulted from the narrow marker coverage. The identified QTL were located on 11 LGs, while 16 LGs had no associations with any QTL for these traits. This could be due to either limited marker coverage or having no detectable QTL on the linkage groups. However, similar results regarding this issue were reported in previous studies (Zhang et al., 2005; Wang et al., 2006; He et al., 2007; Wu et al., 2009).
There were many studies conducted to identify QTL linked to fiber quality traits, using both interspecific (Jiang et al., 1998; Kohel et al., 2001; Paterson et al., 2003; Mei et al., 2004; Lacape et al., 2005; He et al., 2007; Lacape et al., 2009; Zhang et al., 2011), and intraspecific upland cotton maps (Shappley et al., 1998; Ulloa and Meredith, 2000; Zhang et al., 2005; Shen et al., 2005, 2007; Qin et al., 2008; Wu et al., 2009; An et al., 2010). However, there has been no report on QTL mapping of naturally green colored cotton genome in the literature up to now. Besides, the quantification of the individual's cotton fiber colors as seven color parameters using a chromameter device was original in our study. By doing this, we identified 35 QTL for fiber color parameters in total (Table 6).

Table 6. QTL linked to the fiber color parameters

\begin{tabular}{|c|c|c|c|c|}
\hline Fiber color parameter & Marker ID & Linkage Group & LOD & $\mathbf{R}^{2}(\%)^{\dagger}$ \\
\hline \multirow[t]{5}{*}{$L$} & MCACEACA5 & 12 & 3.11 & 14.6 \\
\hline & MCACEACA6 & 12 & 3.11 & 14.6 \\
\hline & MCAAEAAG8 & 15 & 2.33 & 11.1 \\
\hline & MCAAEAAG2 & 20 & 2.05 & 9.8 \\
\hline & MCAGEACC2 & - & 1.79 & 8.7 \\
\hline \multirow[t]{4}{*}{$a$} & MCACEACC2 & 10 & 2.66 & 13 \\
\hline & MCTAEACG2 & 9 & 1.79 & 8.7 \\
\hline & MCTCEAAG1 & 2 & 1.7 & 8.5 \\
\hline & MCAAEACA13 & 18 & 1.67 & 11.3 \\
\hline \multirow[t]{4}{*}{$b$} & MCACEACA7 & 16 & 2.43 & 11.6 \\
\hline & MCAAEACA3 & 16 & 2.39 & 12.1 \\
\hline & MCACEACA8 & 16 & 1.8 & 8.7 \\
\hline & MCATEAGC4 & - & 1.64 & 8.1 \\
\hline \multirow[t]{8}{*}{$\Delta L$} & MCACEACA6 & 12 & 2.99 & 14 \\
\hline & MCACEACA5 & 12 & 2.99 & 14 \\
\hline & MCAAEAAG8 & 15 & 2.4 & 11.4 \\
\hline & MCAAEAAG2 & 20 & 2.17 & 10.4 \\
\hline & MCAGEACC2 & - & 1.82 & 8.8 \\
\hline & MCAAEACA3 & 16 & 1.78 & 9.1 \\
\hline & MCAAEAAG6 & 15 & 1.73 & 8.4 \\
\hline & MCAGEACG3 & 4 & 1.62 & 7.9 \\
\hline \multirow[t]{4}{*}{$\Delta a$} & MCACEACC2 & 10 & 2.7 & 13.2 \\
\hline & MCTAEACG2 & 9 & 1.67 & 8.1 \\
\hline & MCAAEACA13 & 18 & 1.67 & 11.3 \\
\hline & MCTCEAAG1 & 2 & 1.65 & 8.3 \\
\hline \multirow[t]{4}{*}{$\Delta b$} & MCACEACA7 & 16 & 2.43 & 11.6 \\
\hline & MCAAEACA3 & 16 & 2.39 & 12.1 \\
\hline & MCACEACA8 & 16 & 1.79 & 8.7 \\
\hline & MCATEAGC4 & - & 1.62 & 8 \\
\hline \multirow[t]{6}{*}{$\Delta E$} & MCACEACA6 & 12 & 2.51 & 11.9 \\
\hline & MCACEACA5 & 12 & 2.51 & 11.9 \\
\hline & MCAAEACA3 & 16 & 2.27 & 11.6 \\
\hline & MCAAEAAG8 & 15 & 1.87 & 9 \\
\hline & MCAAEAAG2 & 20 & 1.76 & 8.5 \\
\hline & MCAAEAAG6 & 15 & 1.61 & 7.8 \\
\hline
\end{tabular}

${ }^{\dagger} \mathrm{R}^{2}(\%)$ indicates the percentage of the phenotypic variance explained by the QTL. 
We detected five QTL for $L$ indicating color brightness ( $L=0$ is black and $L=100$ is white) on LG12 (2 QTL), 15, 20 and one was not located on any LG. Each QTL (LOD 3.11 ) on LG12 explained $14.6 \%$ of the phenotypic variation. The three QTL can be considered as putative due to $\mathrm{LOD}<2.5$. Four QTL were detected for " $a$ " on LG2, 9, 10 and 18. The fiber color parameter " $a$ ", describes the color range from red to green. The higher the negative " $a$ " value is, the closer the fiber color is to green which is particularly important for our study. The QTL (MCACEACC2) on LG10 with the LOD of 2.66 explained $13 \%$ of the phenotypic variation for " $a$ ". Four QTL were identified for $b$ on LG16 (3 QTL) and one was not located on any LG. Two of the putative QTL for $b$ had $2<\mathrm{LOD}<2.5$. The fiber color parameter $b$ gives yellowblue range, and the positive value is yellow while the negative is blue. The eight QTL were detected for $\Delta L$, showing total black and white color change, on LG12 (2 QTL), 15 (2 QTL), 4, 16, 20, and one was not located on any of the LGs. Each of the two QTL (LOD 2.99) on LG12 explained $14 \%$ of the phenotypic variation. Total red-green color change was described with $\Delta a$. Four QTL were identified for $\Delta a$ on LG2, 9, 10 and 18. The putative QTL (LOD 2.7) explained $13.2 \%$ of the phenotypic variation. All three putative QTL detected for $\Delta b$, describing total yellow-blue color change, were located on LG16, while one other was not located on any LG. Six QTL were detected for $\Delta E$ which defines total color change independent from each other on LG12 (2 QTL), 15 (2 QTL), 16 and 20. The LOD threshold values for the three putative QTL located on LG12 and 16 were in between 2.27 and 2.51. Our QTL results for fiber color parameters were not completely comparable with the other reports in existing literature because currently only two studies were conducted on fiber color yellowness (Paterson et al., 2003), and reflectance (Rd) and yellowness index (+b) (Lacape et al., 2005). Lacape et al. (2005) reported a total of 16 QTL detected for the two color indexes, $\mathrm{Rd}$ and $+\mathrm{b}$, and half of these QTL explained variation observed for both indexes. Paterson et al. (2003) identified a total of 11 QTL for fiber color yellowness but five of these (on the chromosomes 6, 14, 25, and LGA02 and D02) met the permutation based threshold of 3.84. It should also be taken into account that the parental lines, the progenies and the types of the molecular markers used in these studies were different from our study.

In the current study, we detected 4 QTL for fiber length, 2 for fiber uniformity and 2 for fiber elongation (Table 5). Of the 4 QTL, 2 were located on LG1 and one was located on LG8, while the other one was not located on any LG. The two QTL with the highest LOD thresholds of 2.9 and 2.82 on LG1 explained $28.4 \%$ of the phenotypic variation together. Two putative QTL were detected for fiber uniformity, both were not located on any LG and the LOD thresholds were below 2.0. Of these two QTL, one with LOD of 2.48 explained $12.2 \%$ of the phenotypic variation for fiber elongation. However, none were located on any LG. Shen et al. (2007) reported eight QTL for fiber length on A5, A12, D9, D5, D6 (2 QTL), D11, LG3, four QTL for fiber elongation on A3, A11, D8,
LG3, and three QTL for fiber uniformity on D2, D6 and LG2. Lacape et al. (2005) showed that fiber length was associated with chromosomes 12, 14, 20 and 26, while Shen et al. (2005) reported two QTL for fiber length mapped to chromosome 7 and 16. Wu et al. (2009) also identified 4 QTL for fiber length on chromosomes 12, 13, 14, 20 explaining a total phenotypic variation of $38.6 \%$, and 4 QTL for fiber elongation on chromosomes 14, 20, 26 and LG1 with a total phenotypic variation of $28.3 \%$. Ulloa et al. (2000) reported 3 QTL for fiber elongation on LG3 (2 QTL) and LG9 with the phenotypic variations of 3.4, 11.9 and 31.6\%, respectively. Qin et al. (2008) detected one QTL for fiber uniformity on A6. Zhang et al. (2009) identified 4 QTL for fiber length on chromosomes $6,7,8,12$, three QTL for fiber uniformity on chromosomes 6, 7 and 12, and two QTL for fiber elongation on chromosomes 7 and 14. They explained the phenotypic variation between 8.7 and 43.1, 7.4 and 42 , and 7.4 and $38.8 \%$, respectively. However, it is hard to make a complete comparison among these results due to only a few common marker existences among these studies and the differences in maps, which covers different chromosome regions of the cotton genome.

In conclusion, in the present study we obtained individuals having green fiber color and long fiber length, high fiber uniformity and elongation in the $\mathrm{F}_{2}$ population. These recombinant individuals are an asset for future cotton variety studies. Regarding the identification of the QTL controlling important fiber related traits, dominant AFLP markers should be supported with co-dominant markers to acquire more information. However, it is known that all the intraspecific upland cotton maps were characterized by low marker coverage of the genome because molecular marker polymorphism is limited within G. hirsutum (Zhang et al., 2009). Therefore, the number of polymorphic DNA markers within upland cotton maps should be increased in order to detect more QTL or QTL with large effects that will provide a more dense linkage map. The more those commonly informative molecular markers are used in the cotton genome, the greater the likelihood that QTL of interest for fiber color and high fiber quality among various parental lines can be tagged. Such common QTL may be used in MAS breeding to improve fiber quality in naturally colored cotton.

\section{ACKNOWLEDGEMENT}

This project is supported by the Scientific and Technological Research Council of Turkey (Grant No. 1040589)

\section{LITERATURE CITED}

Acikgoz, N., M.E. Akkas, A. Moghaddam, and K. Ozcan. 1994. Turkish data based statistics programmer PC: TARIST. p. 264-267. In Plant Breeding Proc. Turkey's Field Crops Congress-1, Izmir, Turkey. 25-29 April 1994. Ege University, Bornova, Izmir, Turkey.

An, C., J.N. Jenkins, J. Wu, Y. Guo, and J.C. McCarty. 2010. Use of fiber and fuzz mutants to detect QTL for yield components, seed and fiber traits of upland cotton. Euphytica 172:21-34 
Chaudhary, B., J. Singh, S.K. Chopra, A.K. Sharma, M.K. Sharma, and R. Singh. 2010. Genetic analysis for seed cotton yield and fiber quality traits in cotton (Gossypium hirsutum L.). Indian J. Plant Gen. Res. 23(3):292-295.

Creatham, C.L., J.N. Jenkins, C. McCarty, C.E. Watson, and J. $\mathrm{Wu}$. 2003. Genetic variances and combining ability of crosses of American cultivars, Australian cultivars and wilt cottons. J. Cotton Sci. 7:16-22.

Doyle, J.J., and J.L. Doyle. 1987. A rapid DNA isolation procedure for small quantities of fresh leaf tissue. Phytochem. Bull. 19:11-15.

Dutt, Y., X.D. Wang, Y.G. Zhu, and Y.Y. Li. 2004. Breeding for high yield and fiber quality in colored cotton. Plant Breeding 123:145-151.

Endrizzi, J.E., and R.J. Kohel. 1966. Use of telesomes in mapping three chromosomes in cotton. Genetics 54:535-550.

Fan, X.-P., B.-H. Fan, Z.-Q. Xu, and W.-C. Yang. 2009. Inheritance of red leaf and green fiber in cotton and their effects on yield and fiber quality. Cotton Science DOI:CNKI:SUN:MHXB.0.2009-01-007.

Feng, H.J., J. Wang, J.L. Sun, X.Y. Zhang, and Y.H. Jia. 2010. Genetic effects of fiber color in brown cotton (Gossypium hirsutum L.). Acta Agron. Sinica 36(6):961-967.

Fletcher, E. 1907. Mendelian heredity in cotton. J. Agric. Sci. 2:281-282.

Gutiérrez, O.A., S. Basu, S. Saha, J.N. Jenkins, D.B. Shoemaker, C.L. Cheatham, and J.C. McCarty. 2002. Genetic distance among selected cotton genotypes and its relationship with $\mathrm{F}_{2}$ performance. Crop Sci. 42:1841-1847.

He, D.H., Z.X. Lin, X.L. Zhang, Y.C. Nie, X.P. Guo, Y.X. Zhang, and W. Li. 2007. QTL mapping for economic traits based on dense genetic map of cotton with PCR-based markers using the interspecific cross of Gossypium hirsutum vs. Gossypium barbadense. Euphytica 153(1-2):181-197.

Jambhale, N.D., R.M. Paralkar, N.V. Yadav, R.S. Darade, M.D. Yadav, and S.S. Mehetre. 1998. Genetics and improvement of lint characters in naturally colored cotton $(G$. hirsutum L.). p. 66. In New frontiers in cotton research. Proc. World Cotton Res. Conf.-2, Athens, Greece. 6-12 September 1998. Petridis, Thessaloniki, Greece.

Jiang, C.X., R.J. Wright, K.M. El-Zik, and A.H. Paterson. 1998. Polyploid formation created unique avenues for response to selection in Gossypium (Cotton). Proc. Natl. Acad. Sci. 95:4419-4424.

Kohel, R.J., J. Yu, Y.H. Park, and G.R. Lazo. 2001. Molecular mapping and characterization of traits controlling fiber quality in cotton. Euphytica 121:163-172.

Kohel R.J. 1985. Genetic analysis fibre colour variants in cotton. Crop Science 25:793-797.

Kosambi D.D. 1944. The estimation of map distances from recombination values. Ann Eugen 12:172-175.

Lacape, J.M., T.B. Nguyen, B. Courtois, J.L. Belot, M. Giband, J.P. Gourlot, G. Gawrysiak, S. Roques, and B. Hau. 2005. QTL analysis of cotton fiber quality using multiple Gossypium hirsutum x Gossypium barbadense backcross generations. Crop Science 45(1):123-140.

Lacape, J.M., J. Jacobs, T. Arioli, R. Dericker, N. ForestierChiron, D. Llewellyn, J. Jean, E. Thomas, and C. Viot. 2009. A new interspecific, Gossypium hirsutum x G. barbadense, RIL population: towards a unified consensus linkage map of tetraploid cotton. Theor. and Appl. Genetics 119:281-292.

Lander, S., P. Green, J. Abrahamson, A. Barlow, M. Daly, S. Lincoln, and L. Newburg. 1987. MAPMAKER: An interactive computer package for constructing primary genetic linkage maps of experimental and natural populations. Genomics 1:174-181.
Lander, E.S., and D. Botstein. 1989. Mapping Mendelian factors underlying quantitative traits using RFLP linkage maps. Genetics 121:185-199.

Li, D., Y. Nie, and X. Zhang. 2004. Genetic analysis of fiber color on brown upland cotton. J. Huazhong Agric. DOI: CNKI:SUN:HZNY.0.2004-06-003.

Lin, Z., D. He, X. Zhang, Y. Nie, X. Guo, C. Feng, and J.M.C.D. Stewart. 2005. Linkage map construction and mapping QTL for cotton fibre quality using SRAP, SSR and RAPD. Plant Breeding 124:180-187.

Luan, M., X. Guo, Y. Zhang, J. Yao, and W. Chen. 2009. QTL mapping for agronomic and fiber traits using two interspecific chromosome substitution lines of upland cotton. Plant Breeding 128:671-679.

Mei, M., N.H. Syed, W. Gao, P.M. Thaxton, C.W. Smith, D.M. Stelly, and Z.J. Chen. 2004. Genetic mapping and QTL analysis of fiber related traits in cotton (Gossypium). Theor. and Appl. Genetics 108:280-291.

Murthy, M.S.S. 2001. Never say dye: the story of coloured cotton. Resonance 29-35.

Nelson, J.C. 1997. QGENE: software for marker-based genomic analysis and breeding. Mol. Breeding 3:239-245.

Paterson, A.H., Y. Saranga, M. Menz, C. Jiang, and R.J. Wright. 2003. QTL analysis of genotype $x$ environmental interactions affecting cotton fiber quality. Theor. Appl. Genetics 106:384-396.

Price, J.B., X. Cui, and T.A. Calamari. 2001. Assessing the quality of four naturally colored cottons. Textile Res. J. 71(11):993-999.

Qin, H., W. Guo, Y.M. Zhang, and T. Zhang. 2008. QTL mapping of yield and fiber traits based on a four-way cross population in Gossypium hirsutum L. Theor. and Appl. Genetics 117:883-894.

Richmond, T.R. 1943. Inheritance of brown and green lint in upland cotton. J. Am. Soc. Agron. 35:967-975.

Shappley, Z.W., J.N. Jenkins, W.R. Meredith Jr., and J.C. McCarty. 1998. An RFLP linkage map of upland cotton. Theor. and Appl. Genetics 97:756-761.

Shen, X., W. Guo, X. Zhu, Y. Yuan, J.Z. Yu, R.J. Kohel, and T. Zhang. 2005. Molecular mapping of QTL for qualities in three diverse lines in upland cotton using SSR markers. Mol. Breeding. 15:169-181.

Shen, X., W. Guo, Q. Lu, X. Zhu, Y. Yuan, and T. Zhang. 2007. Genetic mapping of quantitative trait loci for fiber quality and yield trait by RIL approach in upland cotton. Euphytica 155:371-380.

Shi, Y.Z., X.M. Du, G.Q. Liu, A.D. Qiang, Z.L. Zhou, Z.E. Pan, and J.L. Sun. 2002. Genetic analysis of naturally colored lint and fuzz of cotton. Acta Gossypii Sinica DOI:CNKI:ISSN:1002-7807.0.2002-04-011.

Silow, R.A. 1944. The inheritance of lint colour in Asiatic cotton. J. Genet. 46:78-115.

Subhan, M., M. Qasim, D.R. Ahmad, M.U. Khan, M.A. Khan, and M.A. Amin. 2003. Combining ability for yield and its component in upland cotton. Asian J. Plant Sci. 2(7):519522.

Ulloa, M., R.G. Cantrell, R.G. Percy, E. Zeiger, and Z. Lu. 2000. QTL analysis of stomatal conductance and relationship to lint yield in an interspecific cotton. J. Cotton Sci. 4:10-18.

Ulloa, M., and W.R. Meredith Jr. 2000. Genetic linkage map and QTL analysis of agronomic and fiber quality traits in an intraspecific population. J. Cotton Sci. 4:161-170.

Ulloa, M., C. Brubaker, and P. Chee. 2007. Cotton. p. 1-49. In C. Kole (ed.) Genome Mapping and Molecular Breeding in Plants. Vol. 6. Technical Crops. Springer-Verlag Berlin Heidelberg, Germany. 
Wang, B., W. Guo, X. Zhu, Y. Wu, N. Huang, and T. Zhang. 2006. QTL mapping of fiber quality in an elite hybrid derived-RIL population of upland cotton. Euphytica 152:367-378.

Wu, J., O.A. Gutierrez, J.N. Jenkins, J.C. McCarty, and J. Zhu. 2009. Quantitative analysis and QTL mapping for agronomic and fiber traits in an RI population of upland cotton. Euphytica 165:231-245.

Wu, S.-X., W. Wang, Z. Li, and X.-Z. Cui. 2010. The color's genetic analysis of brown cotton and the correlation between color and main characters. Hubei Agri. Sci. DOI:CNKI:SUN:HBNY.0.2010-05-014.

Zhan, S., Z. Li, Y. Lin, and Y. Cai. 2008. Quantitative analysis on the inherited characteristics of naturally colored brown cotton fiber color. Chinese Agricultural Science Bulletin DOI:CNKI:SUN:ZNTB.0.2008-12-034.

Zhang, Z.S., Y.H. Xiao, M.H. Luo, X.B. Li, X.Y. Luo, L. Hou, D.M. Li, and Y. Pei. 2005. Construction of a genetic linkage map and QTL analysis of fiber-related traits in upland cotton (Gossypium hirsutum). Euphytica 144:91-99.

Zhang, Z.S., M.C. Hu, J. Zhang, D.J. Liu, J. Zheng, K. Zhang, W. Wang, and Q. Wan. 2009. Construction of a comprehensive PCR-based marker linkage map and QTL mapping for fiber quality traits in upland cotton (Gossypium hirsutum L.). Mol. Breeding 24:49-61.

Zhang Z., J. Rong, V.N. Waghmare, P.W. Chee, O.L. May, R.J. Wright, J.R. Gannaway, and A.H. Paterson. 2011. QTL alleles for improved fiber quality from a wild Hawaiian cotton, Gossypium tomentosum. Theor. Appl. Genet. 123(7):1075-88.

Zhao L., C.P. Cai, T.Z. Zhang, and W.Z. Guo. 2009. Fine mapping of the red plant gene $R 1$ in upland cotton (Gossypium hirsutum). Chinese Sci. Bulletin 54(9):15291533.

Zhu, M., and Z. Wang. 2003. Genetic analysis of cotton colored fiber based on computer assisted identification. Mol. Plant Breeding DOI:CNKI:ISSN:1672-416X.0.2003-01-018. 